\title{
Invited Commentary: Understanding Brain Mechanisms of Pain Processing in Adolescents' Non-Suicidal Self-Injury
}

\author{
Elizabeth Ballard • Abigail Bosk - Maryland Pao
}

Published online: 15 October 2009

(C) US Government 2009

\begin{abstract}
Whereas non-suicidal self injury (NSSI) is reported in 13-23\% of adolescents and is an increasingly studied topic, there has been little investigation into the pathophysiology behind self-injury. This commentary examines recent research into pain and emotional distress to discuss implications for the manner we should understand, research, and treat NSSI in the future. Research indicates that adolescents may be particularly vulnerable to NSSI behaviors due to neurodevelopmental changes in the processing of distress and pain. Additionally, emotional distress and physical pain neural pathways may have been altered in these individuals, leading to the development of NSSI behaviors during adolescence when changes in ongoing brain development may lead to further emotional dysregulation and poor impulse control. Further studies that directly characterize the relationship between emotional distress and physical pain in adolescence, as well as the neural differences between self-injurers and non-selfinjurers, are needed.
\end{abstract}

This paper was prepared by the employees of the US Government making this a "work of the US Government" and therefore copyright is non-transferable. The opinions expressed in the article are the views of the authors and do not necessarily reflect the views of the Department of Health and Human Services or the United States government.

E. Ballard · A. Bosk $\cdot$ M. Pao $(\bowtie)$

National Institute of Mental Health, Clinical Research Center, Building 10, 6-5340, Bethesda, MD 20892-1276, USA

e-mail: paom@mail.nih.gov

E. Ballard

e-mail: ballarde@mail.nih.gov

A. Bosk

e-mail: boska@mail.nih.gov
Keywords Pain · Non-suicidal self-injury $\cdot$ Adolescence

Non-suicidal self-injury (NSSI) is the direct destruction or alteration of body tissue without conscious suicidal intent (Jacobson and Gould 2007; Klonsky 2007; Lloyd-Richardson et al. 2007). NSSI behaviors can range from selfcutting, to burning, self-tattooing and the breaking of bones, with cutting as the most common form of NSSI among adolescents (Briere and Gil 1998; De Leo and Heller 2004). Estimates of recurrent NSSI behaviors in adolescents range from 13-23\% (Jacobson and Gould 2007). Studies show that almost half of community samples of adolescents report some form of self-harm at some point in their lives (Lloyd-Richardson et al. 2007). With the high prevalence rate of NSSI, it is likely that adolescent primary care providers and mental health clinicians will assess and treat these behaviors.

Self-injurers often report that engaging in NSSI helps to regulate intense emotions. Before self-injuring, individuals may feel overwhelmed, frustrated or sad; after injuring, individuals report feeling a sense of relief from pain, calm, dissociation or numbness (Jacobson and Gould 2007; Klonsky 2009; Lloyd-Richardson et al. 2007). For individuals engaging in NSSI, physical injury is believed to serve social (to communicate or elicit a reaction from someone) (Nock 2008), emotional (to regulate emotions) (Nock and Prinstein 2004) and physiological (to decrease physiological arousal in response to emotional distress) functions (Nock and Mendes 2008). Since psychiatric disorders as well as NSSI commonly emerge in adolescence (Kessler et al. 2005), a time of intense and turbulent emotions, the identification and treatment of NSSI in adolescents is particularly important. NSSI is considered by many to be an indication of psychiatric distress and is associated 
with major depressive disorder, obsessive-compulsive disorder, eating disorders and borderline personality disorder (Jacobson and Gould 2007; Nock et al. 2006). By definition, individuals do not report suicidal ideation at the time of injury; however, NSSI is associated with increased risk of later suicide (Nock et al. 2006; Joiner 2005).

While NSSI is an increasingly studied topic, there has been little investigation into the physiological mechanisms behind NSSI, specifically the connections of self-injury to research on the processing of physical pain. NSSI presents researchers and clinicians with a unique opportunity to look at the interaction between distress, emotional dysregulation and physical pain. Clinicians who treat adolescents may be helped through understanding recent neurobiological research concerning self-injury, emotional distress and pain through the lens of adolescent development. What follows is a review of the recent research on pain, NSSI and emotional distress in order to discuss future treatment and research implications in adolescents exhibiting this behavior.

\section{Biological Mechanisms of Physical Pain Processing}

For this commentary, a background on the known biological mechanisms of pain may help to understand NSSI. Pain researchers and clinicians define physical pain as the "unpleasant sensory and emotional experience associated with actual or potential tissue damage" (International Association for the Study of Pain Task Force on Taxonomy 1994). Even within this definition, it is understood that physical pain has both somatosensory and affective (emotional) components. The pain system is unique because of the implications of sensory components in the anterior parietal lobe as well as areas involved in emotion in the limbic system (hippocampus, amygdala, anterior thalamic nuclei and limbic cortex), which mediate mood, sleep, appetite, and endocrine and cardiovascular function.

Painful stimuli are thought to activate multiple brain regions through several ascending pathways from the spinal dorsal horn. These pathways, which activate in parallel as well as serially, include the spinohypothalamic, spinoamygdaloid, and spinothalamic pathways. These and other pathways have projections to specific thalamic nuclei that connect to the anterior cingulate cortex (ACC) and the insular cortex (IC) of the limbic system and also through corticolimbic pathways from the sensory cortex to brain structures involved in other sensory modalities (hearing, smell, and vision) and memory (Price 2000; Klossika et al. 2006). Spinoamygdaloid projections seem to influence fear and avoidance; spinothalamic projections to the parietal lobe identify the location of a pain stimulus while projections to the insular cortex provide information about pain intensity. The processing of pain affect is encoded in the ACC, rather than the somatosensory cortex (Rainville et al. 1997). Projections to the ACC give information about the emotional valence, or unpleasantness, associated with pain. Thus, the somatosensory and affective components of pain perception are processed through several parallel pathways.

Imaging studies have also shown that pain sensation and pain unpleasantness are actually two distinct properties affected by the duration of the stimulus and the presence or absence of assurances of safety (Price 2000). An additional component of pain affect, called "secondary pain affect," refers to the inclusion of memories of pain or imagination about pain in the future (Price 2000), suggesting that contextual information and memory provide cognitive mediation of pain affect. Attention can also modulate pain (Bantick et al. 2002) and exacerbation of pain by anxiety is associated with activity in the hippocampal network (Ploghaus et al. 2001). Thus, cognitive and emotional factors can affect unpleasantness (or pain distress) without changing the intensity of pain (Villemure and Bushnell 2002; Villemure et al. 2003). The ACC, along with the periaqueductal grey (PAG), amygdala, and anterior insula, plays a key role in how mood and pain modulate one another (Weich and Tracey 2009). These areas of the brain are implicated in affective pain processing as separate and distinct from somatosensory pain processing pathways. Understanding distinctions between the somatosensory and affective pain pathways can help explain the physiological mechanisms involved in NSSI, particularly when pain is used to regulate psychological distress.

\section{Pain and NSSI}

Current research on emotional dysregulation as well as somatosensory and affective pain processing in NSSI suggests that adolescents who engage in NSSI may experience heightened emotional distress compared to adolescents who do not engage in NSSI. Adolescents who engage in NSSI may be less able to tolerate emotional distress and may experience higher physiologic reactivity including skin conductance measures in association with stressful events (Deliberto and Nock 2008; Nock and Mendes 2008). These adolescents also have difficulties in social problem solving; when adolescents are given a list of solutions to solve a social problem, adolescents who self injure select more maladaptive responses and report lower self-efficacy for performing adaptive solutions than adolescents who do not self-injure (Deliberto and Nock 2008; Nock and Mendes 2008). Physical pain perception is also altered in adolescents who self injure. In one review of adolescent psychiatric inpatients who engaged in NSSI, the majority reported little to no pain at the time of self-injury (Nock and Prinstein 2005). Changes in distress and pain processing pathways in 
adolescents who do engage in NSSI may provide insight into what motivates adolescents to self-injure.

Recent research with patients with borderline personality disorder (BPD) who self-injure has explored some alterations of pain perception seen in NSSI. Self injuring patients with BPD have higher pain thresholds under both stress and non-stress conditions when compared to healthy controls (Bohus et al. 2000; Schmahl et al. 2008, 2006, 2004). When comparing individuals with BPD who were actively engaging in NSSI with those who had terminated NSSI at least 6 months prior, individuals who were engaging in the behavior were less sensitive to thermal stimuli and laser radiant heat pulses (Ludascher et al. 2009). In particular, pain thresholds significantly increase when individuals with BPD are distressed and have a desire to self-injure (Bohus et al. 2000). Additionally, there is a strong connection between negative emotional arousal and increased pain thresholds in patients with BPD, as well as increased dissociative symptoms (Ludascher et al. 2007). Furthermore, it has also been shown that individuals with BPD who selfinjure have difficulty differentiating between mildly painful and moderately painful hypothetical situations (Russ et al. 1996). Opioid antagonists, such as naltrexone, have not been shown to be an efficacious treatment for NSSI in patients with BPD, suggesting that other mechanisms, in addition to opioid pathways, account for differences in pain perception in NSSI (Russ et al. 1994; Sher and Stanley 2008). Studies in patients with BPD during self-injury suggest pain perception is altered, but targets for treatment have yet to be identified.

Brain imaging studies have shown which brain areas within pain processing pathways may be altered in NSSI. An imaging study with BPD patients showed that the decreased sensation of pain is associated with changes in the system modulating the affective responses to pain, most specifically, the anterior cingulate cortex (ACC) and the amygdala (Schmahl et al. 2006). These results imply that individuals with BPD may have similar physical sensations of pain, but differing emotional reactions. In other words, the affective (rather than somatosensory) processing of pain may be different in individuals who participate in selfharm. Results from these imaging studies show that changes in the ACC and prefrontal cortex (PFC) pathways may be implicated in the altered pain perception in individuals who participate in NSSI.

\section{Social Distress and Pain}

One recently proposed theory, with implications for adolescent NSSI, posits a neural overlap in the processing of pain and social distress. The authors look to the language surrounding social relationships such as "heartache" and "broken heart" as evidence of the overlap between social and physical pain; in short, "why rejection hurts" (Eisenberger 2008; Lieberman and Eisenberger 2009; O'Connor et al. 2008). Specifically, Eisenberger and colleagues suggest that the "unpleasantness" of physical pain is processed in the same area of the brain as "social pain," or distress related to loss, rejection or isolation.

Eisenberger and colleagues have used social exclusion paradigms and recent research on the brain areas involved in the processing of physical pain distress to explore this overlap. For this research, while in the functional magnetic resonance imaging (fMRI) scanner, a participant is led to believe that she is playing a virtual ball-tossing task with other research participants, while in actuality she is participating in a preset program which leads the participant to believe that she is being excluded from most of the gameplaying (Eisenberger et al. 2003). The "social pain" evoked by this task is associated with areas associated with affective pain processing, specifically, increased activation of ACC and decreased activation of PFC in adults (Eisenberger et al. 2003; Eisenberger and Lieberman 2004). Research with healthy adolescents using the same paradigm shows similar patterns of activation in the insula and PFC, but differential activation in the subgenual ACC and ventral striatum, which suggests that adolescents might process social pain and exclusion in a slightly different way than adults (Matsen et al. 2009). Further work in this area has shown that sensitivity to pain is associated with increased sensitivity to social rejection in healthy controls (Eisenberger et al. 2006). Adolescents, then, may process emotional distress and affective pain in similar regions of the brain.

The overlap between affective, physical and social pain processing has implications for adolescent self-injurers. Adolescence is a particularly vulnerable time for social distress and isolation, and the neural system for processing emotional reactions is not fully formed and may have been affected in childhood by negative or traumatic experiences. Therefore, when an adolescent is experiencing emotional distress, the affective processing system of physical pain may be inadvertently activated in order to motivate the individual to seek social support and attachment. Social distress, in the form of feeling isolated or victimized, is also a common reason for NSSI (Hilt et al. 2008). It is possible that, in individuals who engage in NSSI, this pathway is activated to regulate emotions through physical pain, although this paradigm has yet to be tested in adolescent self-injuring populations.

\section{Neurobiological Maturation in Adolescence}

Adolescence is defined as the period of physical and psychological development from the onset of puberty to maturity and is characterized by intense emotions and impulsive and risky behaviors that can lead to serious harm to oneself 
and others (White 2009). Neurobiological maturation in adolescents is characterized by the development of the emotional and cognitive abilities needed for independent functioning throughout adulthood (Yurgelun-Todd 2007). Until recently, it was believed that brain maturation was complete by the beginning of adolescence. However, longitudinal MRI scans of the brains of healthy children (Durston et al. 2001; Lenroot and Giedd 2006) have shown, conclusively, that the brain does not complete maturation by the end of childhood but rather continues to develop throughout adolescence and into young adulthood.

The NIMH longitudinal study of brain development, which began in 1989, has resulted in approximately 5,000 scans from 2,000 subjects, ages 3-30 years, and has led to a greater understanding of brain development in children, adolescents and young adults. The key findings from this study are described below. The prefrontal cortex (PFC), including the frontal lobes, undergo significant remodeling throughout adolescence and young adulthood and this maturatation is correlated with significant behavioral changes in adolescents (Fuster 2002; Gogtay et al. 2004; Lenroot and Giedd 2006; Rosso et al. 2004; Sowell et al. 2004). The frontal lobes play a necessary role in forming memories, directing, movement, controlling emotional urges, planning for the future, and other higher-order cognitive functions needed for survival (White 2009). Frontal lobe gray matter volume (density of neurons) increases throughout childhood and peaks at age 11 for girls and 12 for boys, and then declines throughout the teenage years and into young adulthood (Giedd 2004; Lenroot and Giedd 2006). During adolescence, the brain eliminates synaptic connections that are not used (synaptic pruning), and thus results in a reduction in the volume of frontal gray matter (Ernst and Mueller 2008; White 2009). The maturation of the PFC leads to individuals learning to control their impulses, making plans, and regulating their emotions (Casey et al. 2008; Klenberg et al. 2001; Rosso et al. 2004; Rubia et al. 2000; Tamm et al. 2002; White 2009).

In addition to the frontal lobes, limbic structures such as the amygdala and the nucleus accumbens, modulate adolescent behaviors. The amygdala plays a key role in learning and evoking emotional responses, especially negative emotional responses, to stimuli (White 2009). The nucleus accumbens, which is located within the center of the brain, is the body's key structure in the reward system (Casey et al. 2008). The nucleus accumbens receives neural signals in the form of the neurotransmitter, dopamine. Animal studies have led researchers to believe that the density of dopamine receptors is highest in the nucleus accumbens during adolescence, making this brain region particularly responsive to the reward signals of dopamine (Ernst and Mueller 2008; White 2009). Furthermore, neuroimaging studies have shown that, when making risky choices and processing emotion-laden information, adolescents have larger increases in activity in the amygdala and nucleus accumbens relative to the activity seen in children and adults (Ernst and Mueller 2008). The heightened activity in the amgydala and nucleus accumbens seen in adolescents during emotionally rewarding and risky situations, in combination with underdeveloped behavioral control areas, contributes to adolescents' being prone to engaging in impulsive behavior and making risky decisions (Casey et al. 2008).

Overall, the NIMH longitudinal study of brain development elucidated several structural and neurobiological changes that take place in the brains of children and adolescents. These changes include: (1) childhood peaks of gray matter volume, number of chemical synapses and neurotransmitter receptor density followed by a decrease of all three during adolescence; (2) a linear increase in white matter throughout childhood and adolescence which results in associative cognitive activity due to integration of distant brain regions and; (3) increased reliance on frontal lobe circuitry and decreased reliance on the limbic system and nucleus accumbens (Giedd 2008). These ongoing neuronal changes are believed to influence teenage behavior.

In addition to the structural changes mentioned above, hormonal changes during adolescence are believed to impact the behavior of adolescents. Adolescents display an exaggerated stress response due to an increased release of the steroid hormone cortisol from the hypothalamic-pituitaryadrenal (HPA) axis (Alloy et al. 2006; Walker et al. 2004). During stressful situations, the hypothalamus releases corticotropin-releasing hormone (CRH), which causes the pituitary to release adrenocorticotropic (ACTH), which triggers the adrenal glands to release cortisol. During puberty, there is increased activity in the HPA axis, which results in the increased production and release of cortisol and is thought to contribute to high emotional reactivity of adolescents (White 2009). This increased emotional reactivity and decreased ability to control impulses are two factors that may contribute to engagement in NSSI in adolescence.

Overall, changes in brain structure and function such as those described above are believed to parallel improvements in executive functions such as abstract thought, organization, decision making and planning and response inhibition that are seen throughout adolescence and young adulthood (Rosso et al. 2004; Rubia et al. 2000). However, while these neurobiological changes are occurring, the maturation of the limbic system coupled with immature frontal lobe circuitry, increased dopamine response from the nucleus accumbens and increased activity of the HPA put adolescents at risk for making impulsive decisions, showing heightened emotional reactivity and seeking immediate rewards rather than thinking about long term consequences. Perturbations in these neurobiologic systems may contribute to the expression of NSSI and psychiatric disorders in adolescents. 


\section{Early Life Stress and Pain Perception}

The overlap between emotional distress and pain processing has also been explored in recent research on early life stress and trauma. It is known that early life experiences of trauma affects the neural pathways related to emotional distress processing (Blaise et al. 2008; Gunnar and Fisher 2006; McEwen 2003). The effect of stress on neuronal pathways is largely dependent on the developmental stage of the individual at the time of the trauma. For example, stress and trauma during birth to 2 years may particularly affect the developing hippocampus, while stress during early childhood may have particular implications for amygdala volume, and stress during adolescence may impact the development of the frontal lobes (Lupien et al. 2009). Adolescence may be a particularly sensitive period for stress to affect the development of depressive behaviors (Leussis and Andersen 2008) as well as the emergence of other psychiatric disorders (Kessler et al. 2005), suggesting that early life events have an impact later in life on the brain regions related to emotional processing.

However, in addition to emotional distress processing, early life experiences can also affect later pain perception and processing. Repeated early-life stress, particularly physically painful experiences, may alter pain perception (Nijenhuis et al. 1998). Adults who were sexually abused as children report increased reports of pain, independent of depression (Brown et al. 2005). Studies of individuals who were abused show alterations in areas of the brain associated with affective pain processing. In a review of the literature, childhood abuse and trauma is associated with reduced size of ACC, PFC, left cerebral cortex, hippocampus, amygdala and corpus callosum (Teicher et al. 2002). Additionally, individuals with a history of physical and/or sexual abuse have increased activation in the posterior and middle dorsal cingulate cortex (Ringel et al. 2008), suggesting that early life experiences can impact the affective processing of pain.

In sum, early-life stressors, such as physical or sexual abuse, affects neural pathways, both in the processing of emotional distress and dysregulation as well as those implicated in the processing of affective pain. It is possible that the perception of pain in individuals who engage in NSSI is affected by previous experiences of abuse or other negative early-life experiences although these risk factors are not necessarily causal (Klonsky and Moyer 2008). Risk factors for NSSI include history of abuse and negative life events (Jacobson and Gould 2007; Weierich and Nock 2008). The impact of early life experiences on both emotional dysregulation and pain may lead to the use of pathways involved in processing of physical pain to regulate emotions, particularly in adolescents who are vulnerable to developmental emotional lability.
Limitations and Implications of the Current Research

There are limitations to drawing from different fields of research, such as pain, emotional regulation, and brain imaging research to supplement the current state of research on NSSI in adolescents. One limitation of this research is the emphasis on borderline personality disorder as a proxy for NSSI in the larger literature. Also, many of the studies that examine pain in the context of self-injury use adult, rather than adolescent, patients for research participants. Going forward, more research is warranted with NSSI populations that are not necessarily borderline or female. Another limitation includes the need to move from theoretical hypothesis testing to collaborative research with clinicians. Adolescent self-injurers should be studied, as this behavior most often occurs in this population. Further work in this area could lead to more specific treatments that incorporate new research about pain and emotion regulation to target (and prevent) these behaviors.

When working with adolescents who engage in NSSI, clinicians may also consider past experiences of pain (through a detailed pain history) as a factor in self-injury (not just that these patients are suicidal or doing it for attention) when working with NSSI in their practice. A history of severe or prolonged stress or physical/sexual abuse may change a person's biology during developmentally vulnerable periods and have implications for emergent NSSI.

\section{Conclusions}

NSSI is a unique behavior to investigate the interaction between distress and pain. Since this behavior appears to be most common during adolescence, a neurodevelopmental perspective is needed in researching and treating these youth to see how these self-injuring behaviors develop and are maintained. Adolescents may be particularly vulnerable to emotional reactivity and altered decision making due to their stage in brain development. Alterations in the affective aspects of acute physical pain pathways may have implications for how emotional distress is managed in patients who engage in NSSI. The overlap in the processing of physical pain and emotional distress may be a potential area of further study for this population.

Acknowledgments This research was supported by the Intramural Research Program of the National Institutes of Health and the National Institutes of Mental Health.

\section{References}

Alloy, L. B., Abramson, L. Y., Waishaw, P. D., Keyser, J., \& Gerstein, R. K. (2006). A cognitive vulnerability-stress perspective on bipolar spectrum disorders in a normative adolescence 
brain, cognitive and emotional development context. Developmental Psychopathology, 18, 1055-1103.

Arntz, A., \& Claassens, L. (2004). The meaning of pain influences its experienced intensity. Pain, 109, 20-25.

Bantick, S. J., Wise, R. G., Ploghaus, A., Clare, S., Smith, S. M., \& Tracey, I. (2002). Imaging how attention modulates pain in humans using functional MRI. Brain, 125, 310-319.

Blaise, J. H., Koranda, J. L., Chow, U., Haines, K. E., \& Dorward, E. C. (2008). Neonatal isolation stress alters bidirectional long-term synaptic plasticity in amygdalo-hippocampal synapses in freely behaving adult rats. Brain Research, 1193, 25-33.

Bohus, M., Limberger, M., Ebner, U., Glocker, F. X., Schwarz, B., Wernz, M., et al. (2000). Pain perception during self-reported distress and calmness in patients with borderline personality disorder and selfmutilating behavior. Psychiatry Research, 95, 251-260.

Braid, L., \& Cahusac, P. (2006). Decreased sensitivity to self-inflicted pain. Pain, 124, 134-139.

Briere, J., \& Gil, E. (1998). Self-mutilation in clinical and general population samples: Prevalence, correlates, and functions. American Journal of Orthopsychiatry, 68, 609-620.

Brown, J., Berenson, K., \& Cohen, P. (2005). Documented and selfreported child abuse and adult pain in a community sample. Clinical Journal of Pain, 21, 374-377.

Casey, B. J., Getz, S., \& Galvan, A. (2008). The adolescent brain. Developmental Review, 28, 62-77.

De Leo, D., \& Heller, T. S. (2004). Who are the kids who self-harm? An Australian self-report school survey. Medical Journal of Australia, 181, 140-144.

Deliberto, T. L., \& Nock, M. K. (2008). An exploratory study of correlates, onset, and offset of non-suicidal self-injury. Archives of Suicide Research, 12, 219-231.

Durston, S., Hulshoff Pol, H. E., Casey, B. J., Giedd, J. N., Buitelaar, J. K., \& van Engeland, H. (2001). Anatomical MRI of the developing human brain: What have we learned? Journal of the American Academy of Child and Adolescent Psychiatry, 40, $1012-1020$.

Eisenberger, N. I. (2006). Identifying the neural correlates underlying social pain: Implications for developmental processes. Human Development, 49, 273-293.

Eisenberger, N. (2008). Understanding the moderators of physical and emotional pain: A neural systems-based approach. Psychological Inquiry, 19, 189-195.

Eisenberger, N. I., Jarcho, J. M., Lieberman, M. D., \& Naliboff, B. D. (2006). An experimental study of shared sensitivity to physical pain and social rejection. Pain, 126, 132-138.

Eisenberger, N. I., \& Lieberman, M. D. (2004). Why rejection hurts: A common neural alarm system for physical and social pain. Trends in Cognitive Science, 8, 294-300.

Eisenberger, N. I., Lieberman, M. D., \& Williams, K. D. (2003). Does rejection hurt? An fMRI study of social exclusion. Science, 302, 290-292.

Ernst, M., \& Mueller, S. C. (2008). The adolescent brain: Insights from functional neuroimaging research. Developmental Neurobiology, 68, 729-743.

Fuster, J. M. (2002). Frontal lobe and cognitive development. Journal of Neurocytology, 31, 373-385.

Giedd, J. N. (2004). Structural magnetic resonance imaging of the adolescent brain. Annals of the New York Academy of Sciences, $1021,77-85$

Giedd, J. N. (2008). The teen brain: Insights from neuroimaging. The Journal of Adolescent Health, 242, 335-343.

Gogtay, N., Giedd, J. N., Lusk, L., Hayashi, K. M., Greenstein, D., \& Vaituzis, A. C. (2004). Dynamic mapping of human cortical development during childhood through early adulthood. Proceedings of the National Academy of Sciences of the United States of America, 101, 263-271.
Gunnar, M. R., \& Fisher, P. A. (2006). Bringing basic research on early experience and stress neurobiology to bear on preventative interventions for neglected and maltreated children. Development and Psychopathology, 18, 651-677.

Hilt, L. M., Cha, C. B., \& Nolen-Hoeksema, S. (2008). Nonsuicidal self-injury in young adolescent girls: Moderators of the distressfunction relationship. Journal of Consulting and Clinical Psychology, 76, 63-71.

International Association for the Study of Pain Task Force on Taxonomy. (1994). Classification of chronic pain: Description of chronic pain syndromes and definition of pain terms (2nd ed.) IASP Press.

Jacobson, C. M., \& Gould, M. (2007). The epidemiology and phenomenology of non-suicidal self-injurious behavior among adolescents: A critical review of the literature. Archives of Suicide Research, 11, 129-147.

Joiner, T. (2005). Why people die by suicide. Cambridge: Harvard University Press.

Kaufman, J., \& Charney, D. (2001). Effects of early stress on brain structures and function: Implications for understanding the relationship between child maltreatment and depression. Development and Psychopathology, 13, 451-471.

Kessler, R. C., Berglund, P., Demler, O., Jin, R., Merikangas, K. R., \& Walters, E. E. (2005). Lifetime prevalence and age-of-onset distributions of DSM-IV disorders in the National comorbidity survey replication. Archives of General Psychiatry, 62, 593-602.

Klenberg, L., Korkman, M., \& Lahti-Niittila, P. (2001). Differential development of attention and executive functions in 3- to 12year-old finnish children. Developmental Neuropsychology, 20, 407-428.

Klonsky, E. D. (2007). The functions of deliberate self-injury: A review of the evidence. Clinical Psychology Review, 27, 226239.

Klonsky, E. D. (2009). The functions of self-injury in young adults who cut themselves: Clarifying the evidence for affect-regulation. Psychiatry Research, 166, 260-268.

Klonsky, E. D., \& Moyer, A. (2008). Childhood sexual abuse and non-suicidal self-injury: Meta analysis. British Journal of Psychiatry, 192, 166-170.

Klossika, I., Flor, H., Kamping, S., Bleichhardt, G., Trautmann, N., Treede, R. D., et al. (2006). Emotional modulation of pain: A clinical perspective. Pain, 124, 264-268.

Lenroot, R. K., \& Giedd, J. N. (2006). Brain development in children and adolescents: Insights from anatomical magnetic resonance imaging. Neuroscience and Biobehavioral Review, 30, 718-729.

Leussis, M. P., \& Andersen, S. L. (2008). Is adolescence a sensitive period for depression? Behavioral and neuroanatomical findings from a social stress model. Synapse, 62, 22-30.

Lieberman, M. D., \& Eisenberger, N. I. (2009). Pains and pleasures of social life. Science, 323, 890-891.

Lloyd-Richardson, E. E., Perrine, N., Dierker, L., \& Kelly, M. L. (2007). Characteristics and functions of non-suicidal self-injury in a community sample of adolescents. Psychological Medicine, 37, 1183-1192.

Lorenz, J., Minoshima, S., \& Casey, K. L. (2003). Keeping pain out of mind: The role of the dorsolateral prefrontal cortex in pain modulation. Brain, 126, 1079-1091.

Ludascher, P., Bohus, M., Lieb, K., Philipsen, A., Jochims, A., \& Schmahl, C. (2007). Elevated pain thresholds correlate with dissociation and aversive arousal in patients with borderline personality disorder. Psychiatry Research, 149, 291-296.

Ludascher, P., Greffrath, W., Schmahl, C., Kleindienst, N., Kraus, A., Baumgartner, U., et al. (2009). A cross-sectional investigation of discontinuation of self-injury and normalizing pain perception in patients with borderline personality disorder. Acta Psychiatrica Scandinavica, 120, 62-70. 
Lupien, S. J., McEwen, B. S., Gunnar, M. R., \& Heim, C. (2009). Effects of stress throughout the lifespan on the brain, behaviour and cognition. Nature Reviews Neuroscience, 10, 435-445.

Matsen, C. L., Eisenberger, N. I., Borofsky, L. A., Pfeifer, J. H., McNealy, K., Mazziota, J. C., et al. (2009). Neural correlates of social exclusion during adolescence: Understanding the distress of peer rejection. Scan, 4, 143-157.

McEwen, B. S. (2003). Early life influences on life-long patterns of behavior and health. Mental Retardation and Developmental Disabilities, 9, 149-154.

Nemoto, H., Toda, H., Nakajima, T., Hosokawa, S., Okada, Y., Tamamoto, K., et al. (2003). Fluvoxamine modulates pain sensation and affective processing of pain in human brain. Neuroreport, 13, 791-797.

Nijenhuis, E. R., Vanderlinden, J., \& Spinhoven, P. (1998). Animal defensive reactions as a model for trauma-induced dissociative reactions. Journal of Traumatic Stress, 11, 243-404.

Nock, M. K. (2008). Actions speak louder than words: An elaborated theoretical model of the social functions of self-injury and other harmful behaviors. Applied and Preventative Psychology, 12, 159-168.

Nock, M. K., Joiner, T. E., Gordon, K. H., Lloyd-Richardson, E., \& Prinstein, M. J. (2006). Non-suicidal self-injury among adolescents: Diagnostic correlates and relation to suicide attempts. Psychiatry Research, 144, 65-72.

Nock, M. K., \& Mendes, W. B. (2008). Physiological arousal, distress tolerance, and social problem-solving deficits among adolescent self-injurers. Journal of Consulting and Clinical Psychology, 76, $28-38$.

Nock, M. K., \& Prinstein, M. J. (2004). A functional approach to the assessment of self-mutilative behavior. Journal of Consulting and Clinical Psychology, 72, 885-890.

Nock, M. K., \& Prinstein, M. J. (2005). Contextual features and behavioral functions of self-mutilation among adolescents. Journal of Abnormal Psychology, 114, 140-146.

O'Connor, M.-F., Wellisch, D. K., Stanton, A. L., Eisenberger, N. I., Irwin, M. R., \& Lieberman, M. D. (2008). Craving love? Enduring grief activates brain's reward center. NeuroImage, 42, 969-972.

Ploghaus, A., Narain, C., Beckmann, C. F., Clare, S., Bantick, S., Wise, R., et al. (2001). Exacerbation of pain by anxiety is associated with activity in a hippocampal network. The Journal of Neuroscience, 21, 9896-9903.

Price, D. D. (2000). Psychological and neural mechanisms of the affective dimension of pain. Science, 288, 1769-1772.

Rainville, P., Duncan, G. H., Price, D. D., Carrier, B., \& Bushnell, M. C. (1997). Pain affect encoded in human anterior cingulate but not somatosensory cortex. Science, 277, 968-971.

Ringel, Y., Drossman, D. A., Leserman, J. L., Suyenobu, B. Y., Wilber, K., Lin, W., et al. (2008). Effect of abuse history on pain reports and brain responses to aversive visceral stimulation: An fMRI study. Gastroenterology, 134, 396-404.

Rosso, I. M., Young, A. D., Femia, L. A., \& Yurgelun-Todd, D. A. (2004). Cognitive and emotional components of frontal lobe functioning in childhood and adolescence. Annals of the New York Academy of Sciences, 1021, 355-362.

Rubia, K., Overmeyer, S., Taylor, E., Brammer, M., Williams, S. C., Simmons, A., et al. (2000). Functional frontalisation with age: Mapping neurodevelopmental trajectories with fMRI. Neuroscience and Biobehavioral Review, 24, 13-19.

Russ, M. J., Clark, W. C., Cross, L. W., Kemperman, I., Kakuma, T., \& Harrison, K. (1996). Pain and self-injury in borderline patients: Sensory decision theory, coping strategies, and locus of control. Psychiatry Research, 63, 57-65.

Russ, M. J., Roth, S. D., Kakuma, T., Harrison, K., \& Hull, J. W. (1994). Pain perception in self-injurious borderline patients: Naloxone effects. Biological Psychiatry, 35, 207-209.
Salomons, T. V., Johnstone, T., Backonja, M. M., \& Davidson, R. J. (2004). Perceived controllability modulates the neural response to pain. The Journal of Neuroscience, 24, 7199-7203.

Schmahl, C., Bohus, M., Esposito, F., Treede, R., Di Salle, F., Greffrath, W., et al. (2006). Neural correlates of antinociception in borderline personality disorder. Archives of General Psychiatry, 63, 659-667.

Schmahl, C., Greffrath, W., Baumgartner, U., Schlereth, T., Magerl, W., Philipsen, A., et al. (2004). Differential nociceptive deficits in patients with borderline personality disorder and self-injurious behavior: Laser-evoked potentials, spatial discrimination of noxious stimuli, and pain ratings. Pain, 110, 470-479.

Schmahl, C., Meinzer, M., Zeuch, A., Fichter, M., Cebulla, M., Kleindienst, N., Ludascher, P., Steil, R., \& Bohus, M. (2008). Pain sensitivity is reduced in borderline personality disorder, but not in posttraumatic stress disorder and bulimia nervosa. The World Journal of Biological Psychiatric, published ahead of print.

Sher, L., \& Stanley, B. H. (2008). The role of endogenous opiods in the pathophysiology of self-injurious and suicidal behavior. Archives of Suicide Research, 12, 299-308.

Smith, W. B., Gracely, R. H., \& Safer, M. A. (1998). The meaning of pain: Cancer patients' rating and recall of pain intensity and affect. Pain, 78, 123-129.

Sowell, E. R., Thompson, P. M., \& Toga, A. W. (2004). Mapping changes in the human cortex throughout the span of life. Neuroscientist, 10, 372-392.

Tamm, L., Menon, V., \& Reiss, A. L. (2002). Maturation of brain function associated with response inhibition. Journal of the American Academy of Child and Adolescent Psychiatry, 41, 1231-1238.

Teicher, M. H., Andersen, S. L., Polcari, A., Anderson, C. M., Navalta, C. P., \& Kim, D. M. (2002). The neurobiological consequences of early stress and childhood maltreatment. Neuroscience and Biobehavioral Reviews, 27, 33-44.

Villemure, C., \& Bushnell, M. C. (2002). Cognitive modulation of pain: How do attention and emotion influence pain processing? Pain, 95, 195-199.

Villemure, C., Slotnick, B. M., \& Bushnell, M. C. (2003). Effects of odors on pain perception: Deciphering the roles of emotion and attention. Pain, 106, 101-108.

Wager, T. D., Rilling, J. K., Smith, E. E., Sokolik, A., Casey, K. L., Davidson, R. J., et al. (2004). Placebo-induced changes in fMRI in the anticipation and experience of pain. Science, 303, 1162-1167.

Walker, E. F., Sabuwalla, Z., \& Huot, R. (2004). Pubertal neuromaturation, stress sensitivity, and psychopathology. Developmental Psychopathology, 16, 807-824.

Weich, K., \& Tracey, I. (2009). The influence of negative emotions on pain: Behavioral effects and neural mechanisms. NeuroImage, 47, 987-994.

Weierich, M. R., \& Nock, M. K. (2008). Posttraumatic stress symptoms mediate the relation between childhood sexual abuse and nonsuicidal self-injury. Journal of Consulting and Clinical Psychology, 76, 39-44.

White, A. M. (2009). Understanding adolescent brain development and its implications for the clinician. Adolescent Medicine, 20, 73-90.

Yurgelun-Todd, D. (2007). Emotional and cognitive changes during adolescence. Current Opinion in Neurobiology, 17, 251-257.

\section{Author Biographies}

Elizabeth Ballard is a predoctoral fellow at the National Institute of Mental Health and a doctoral student at the Catholic University of America. Her major research interests include emotional dysregulation in adolescents and suicide prevention. 
Abigail Bosk is a postbaccalaurate fellow at the National Institute of Mental Health. Her research interests are in adolescent development and chronic illness.

Maryland Pao is the Clinical Director of the National Institute of Mental Health. She received her M.D. from Johns Hopkins Medical
School and is board certified in general and child and adolescent psychiatry as well as psychosomatic medicine. Her major research interest is the effects of chronic illness and physical pain on biological and emotional development. 